\title{
El método natural de Piferrer: un pionero en la apuesta por la enseñanza de la pronunciación inglesa ${ }^{1}$
}

\author{
JaVier Villoria Prieto \\ Universidad de Granada
}

Recibido: 12 Abril 2007 / Aceptado: 4 Mayo 2007

ISSN: $1697-7467$

He that knows no other tongue knows little of his own.

(Göethe)

RESUMEN: El presente trabajo, un estudio histórico descriptivo, es parte de un ambicioso proyecto que pretende conocer y analizar la metodología que se ha seguido en la enseñanza-aprendizaje de la pronunciación inglesa en España, desde sus orígenes hasta nuestros días. Se realiza a través de los libros escolares que se utilizaron en las aulas. $\mathrm{Y}$ es que los libros de textos son una excelente fuente de información para conocer la Historia de la Educación y, en nuestro caso particular, la historia de la enseñanza de la pronunciación inglesa. Ciertamente, estos materiales han sido muy poco empleados en la investigación. Para realizar este estudio nos servimos de la obra del maestro de lenguas catalán Francisco Piferrer y estudiamos sus técnicas de transcripción, su diseño de la fonética y la metodología de la enseñanza de la pronunciación que utilizaba.

Palabras clave: metodología, Educación, enseñanza de la pronunciación, métodos de enseñanza.

\begin{abstract}
This paper, a historical descriptive study, is part of a ambitious reserach project that aims to know and analyse the methodology displayed in the teaching and learning process of English in the Spanish educational system from its origin up to the present time. It is carried out through textbooks that were used in the classrooms. We consider them a fundamental source to know the history of Eduation, in our case the history of the teaching of English pronunciation in Spain. These materials have been scarcely used in research, and our pourpose is to make the most of the potential they held. To carry out this study we use the work of the catalan teacher of languages Francisco Piferrer and analyse his transcription techniques, phonetic design and the methodology he used to teach the English pronunciation.
\end{abstract}

Key words: methodology, Education, pronunciation teaching, teaching methods.

1 Este trabajo viene a complementar un estudio histórico descriptivo longitudinal para analizar qué metodología se ha seguido en la enseñanza de la pronunciación inglesa en España desde sus orígenes hasta nuestros días. Para llevarlo a cabo nos vamos a centrar en los libros escolares que se utilizaron en las aulas con un triple objetivo: obtener una visión general y longitudinal de cómo se ha enseñado la fonética inglesa en España; identificar, describir y analizar los distintos métodos empleados y, finalmente, tratar de comparar los utilizados entonces y los que hoy día se aplican. 


\section{INTRODUCCIÓN}

Este trabajo pretende arrojar algo de luz sobre el desasistido campo de la enseñanza y aprendizaje del inglés como lengua extranjera en la España del siglo XIX. Pero este lamento de Mar Vilar (2004) tiene aún más de dramático cuando intentamos descubrir no ya el papel que jugó el aprendizaje y enseñanza de la lengua inglesa en la España decimonónica sino conocer la metodología o la didáctica de la enseñanza de su pronunciación.

Con la obra del catalán Francisco Piferrer emprendemos un proyecto que busca conocer los métodos de enseñanza de la pronunciación inglesa para intentar descubrir las causas que tan tozudamente reinciden en la pobreza de la competencia comunicativa oral de los discentes españoles como sistemáticamente indica el informe Pisa. Para ello nos retrotraemos al siglo XIX. El motivo es que sabemos que desde finales del siglo XVII hasta principios del XX fue el francés la lengua que se estudiaba y empleaba en los foros político-diplomáticos, culturales y económicos. Mas, poco a poco la lengua inglesa fue ganando interés y relevancia, aunque realmente apenas sabemos nada del momento en que la enseñanza de la lengua inglesa empieza a ganar adeptos en el campo de enseñanza-aprendizaje de las lenguas extranjeras. Viña Rouco (2005) ofrece algunas pistas de su introducción "en colegios de primera y segunda enseñanza como enseñanzas de adorno". Creemos que a través del estudio y análisis en profundidad de las obras que a tal fin se empleaban, diccionarios, gramáticas, crestomatías, métodos, vademecuns, etc, nos ofrecerán la información que necesitamos. En este estudio, analizamos la obra Método Natural del maestro de lenguas catalán Francisco Piferrer. Trazamos inicialmente unos rasgos biográficos para conocer al personaje, para, a continuación, estudiar su obra y, dentro de ésta, nos centrarnos en lo que más nos interesa: su diseño de la fonética, técnicas de trascripción y metodología que empleaba para enseñar a pronunciar correctamente la lengua de Shakespeare.

\section{Francisco Piferrer}

Era natural de Lloret de Mar, provincia de Gerona. Había nacido el 15 de marzo de 1813 en el seno de una familia ilustrada, con medios económicos. Cursó latín en Blanes, retórica en Olot y filosofía en Gerona. Después de haber desempeñado una cátedra de latinidad, ganada por oposición, pasó a Francia, donde se dedicó al estudio de la jurisprudencia y de lenguas antiguas y modernas ${ }^{2}$. Dos líneas de texto, impresas bajo su nombre en la portada de la obra con la que pretende iniciar la enseñanza del inglés a los españoles, le sirven para presentar sus credenciales. En ellas deja constancia de sus títulos, saberes y experiencia en el campo de la enseñanza de idiomas modernos. Afirma que era maestro de lenguas (conocía el francés, el inglés, el español y el catalán), autor de obras filológicas (el Tableau de la Littérature espagnole, El idioma francés puesto al alcance de todos y El idioma Inglés puesto al alcance de todos), de filosofía (El ser y no ser), y de literatura e historia (Nobiliario de

${ }^{2}$ Manuel Ovilo y Otero: Manual de Biografía y Bibliografía de los escritores del siglo XIX. Tomo II. París: Librería de Rosa y Bouret, 1859. 
los reinos y señoríos de España, una de las más importantes obras del siglo XIX). Había estudiado en la Universidad de París, de la que era bachiller en letras. De regreso a España se estableció en Madrid, donde dio relevantes pruebas de laboriosidad y aplicación al estudio. En 1847 ejercía de regente de la Universidad de Madrid, en la que impartía lenguas. Parece ser que era conocido y que contaba con muy buenas amistades y relaciones en el mundo editorial europeo. De ello dan fe las referencias a los lugares en los que podía adquirirse su última obra, El Método natural, como era conocida en España. En Madrid, a parte de la imprenta editora de Monier, en su residencia particular de la calle de Victoria, número 2. En Barcelona, en la librería familiar. En París, en la librería internacional de Baudry, quai Malaquai, número 3. En Toulouse, en el establecimiento de los señores Bon y Privat. Y en todas las principales librerías de España, Francia e Inglaterra.

La obra que pretendemos estudiar fue impresa en Madrid en 1847, en los talleres que la imprenta-librería de Casimiro Monier tenía en la Carrera de San Jerónimo. Como era costumbre en aquellos tiempos, llevaba un extenso título en el que el autor quería dejar constancia de sus fines y propósitos: El Idioma Inglés puesto al alcance de todos o Método Natural para aprender el inglés de un modo fácil y agradable, sin cansar la memoria. Empresa nada fácil, que él procurará sea tarea agradable y sencilla, pensando siempre en aquellos que hacen el esfuerzo de estudiarlo.

\section{RAZONES PARA ESCRIBIR ESTA GRAMÁtiCa}

La obra de Piferrer cuenta con un prólogo relativamente breve, fechado el 15 de febrero de 1847. En él el autor expresa, entre otras, dos ideas fundamentales y novedosas para aquel tiempo. Ideas que resultan claves para interpretar su trabajo lingüístico. Una, las razones de por qué escribió esta obra y cómo quería que fuera de utilidad para los españoles. Otra, las bases lingüísticas del método que él piensa seguir para enseñar el inglés y la fonética, alejadas de los caminos que en esos momentos seguían en Europa los gramáticos italianos, alemanes, franceses e ingleses en sus academias de lenguas y en sus textos de aprendizaje impresos.

Por lo que se refiere a la primera idea, expone una teoría innovadora para aquellos años: el aprendizaje de idiomas como medio de recuperar la grandeza y el esplendor político de España. Con su trabajo pretende rescatar el prestigio perdido de los tiempos de Carlos V y Felipe II, "cuando el sol no se ponía en las provincias de España y cuando ésta estaba al frente de la civilización europea. Entonces, todas las demás naciones se esforzaban en seguir sus huellas, en adoptar sus costumbres, haciendo alarde de hablar su idioma las cortes de Baviera, de Austria, de Francia y muchas otras"3. Insiste Piferrer en que de nada sirve, ni tiene remedio alguno, lamentarse de pasadas glorias, poderes y felicidades, y se pregunta si esa situación de gloria y brillantez ha desaparecido para siempre y si no existe esperanza alguna de recobrar la antigua preponderancia de nuestros abuelos. La respuesta es rotunda: España no puede desistir de seguir ocupando un lugar preferente al lado de las más impor-

${ }^{3}$ Francisco Piferrer (1847): El idioma inglés puesto al alcance de todos o Método natural. Madrid: Librería de Casimiro Monier, p.5. 
tantes naciones y de influir de un modo poderoso en los destinos de los demás países. Lograrlo es cuestión de tiempo, de esfuerzo en común, y de que cada uno trabaje según sus posibilidades. "Y de igual manera que se mudaron los tiempos, dice el autor, modifíquense también las ideas"4. En esos momentos hay dos naciones que rivalizan en gloria y poder: Francia e Inglaterra. Lo que hay que hacer es estudiar los elementos que constituyen su prosperidad y grandeza; aprovechar las lecciones de unión, de actividad física e intelectual, de comercio e industria que nos están dando, y hacer nuestras sus costumbres e idiomas, como en otros tiempos hicieron ellos con el nuestro. Y, si no es posible lograr los niveles de Inglaterra y Francia, al menos hay que conseguir alcanzar la posición que a España corresponde. Francisco Piferrer se propone como meta de sus esfuerzos al publicar estas dos obras para aprender el francés y el inglés, tres objetivos: el primero, animar a los españoles a familiarizarse con los idiomas francés e inglés; el segundo, allanarles, en cuanto esté en sus manos, las dificultades que ofrecen estudios de esa clase y, finalmente, mostrarles lo injusto que resulta el desdén con que estas dos naciones miran nuestra lengua.

La segunda idea (métodos y caminos a seguir para el estudio del inglés) es la parte más atractiva y nueva que presenta Piferrer. Parte de un proceso de reflexión en el que constata los pocos adelantos que en el aprendizaje de este idioma están consiguiendo los españoles. Afirma que son muchas las gramáticas existentes en el mercado editorial, como numerosos son los maestros de lenguas. Los aficionados a su estudio también son muchos, pero es notable los escasos resultados que se están consiguiendo. Concluye su reflexión con estas palabras: "es escasísimo el número de los que llegan, no diré a hablar, mas ni aun a leer medianamente el francés o el inglés. Hace ya muchos años que tan extraña anomalía llamó mi atención, induciéndome naturalmente a investigar la causa de tan desagradable resultado"s.

Con el único fin de ser útil a los estudiosos españoles y remediar en lo posible los poquísimos resultados que se estaban consiguiendo, se decidió a apartarse en sus planteamientos y metodología del camino común y trillado que seguían los maestros de lenguas europeos en la enseñanza de los idiomas modernos. Se propuso como meta de su proyecto la actitud de los romanos frente al aprendizaje del griego. Aquellos consiguieron hablar y escribir el griego con tanta pureza y facilidad como los mismos atenienses. Analizó Piferrer los medios de que se valían para estudiar dicha lengua con tal éxito. Pronto llegó a la conclusión de que su método era muy diferente del que se sigue en nuestros días para estudios análogos. Los romanos estaban persuadidos de que una lengua no era un arte que pudiera aprenderse con reglas y teoría. Se esmeraban en adquirirla primero con la práctica, antes de pensar en estudiar su mecanismo interno. Y así, "en lugar de comenzar, como se hace entre nosotros, por la aplicación árida de las reglas gramaticales, comenzaban por la lectura

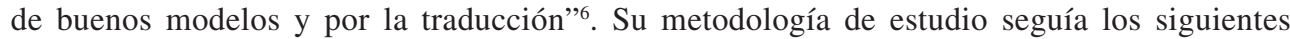
estadios: leían trozos selectos, los traducían, cotejaban el texto con la traducción y ésta con aquél, escribían luego dichos trozos repetidas veces hasta que pudiesen reproducirlos con sólo mirar la traducción, corroborando al fin estos conocimientos prácticos con una sencilla ex-

4 Ibídem, p.6.

5 Ibídem.

6 Ibídem. 
plicación de las reglas más esenciales. Ése era el método que seguían los romanos para familiarizarse con el griego, y ése será el que Francisco Piferrer se propone plantear en España. Era consciente de las dificultades que entrañaba su método, porque "no se me esconde cuán poderosa es la costumbre, conozco cuán fuerte es el yugo que con frecuencia ella impone al hombre, no permitiéndole hacer uso de la razón, y preveo por consiguiente la indiferencia y tal vez el desagrado con que al principio los señores profesores verán un trabajo que sólo se dirige a dar realce y atractivo al estudio de lenguas, haciendo que las lecciones sean menos fastidiosas, menos difíciles y más provechosas. Pero sean cuales fueren los objetivos y dificultades que tenga que superar, íntimamente convencido de la bondad de mis tareas, no perdonaré ningún esfuerzo ni sacrificio para darle feliz cima, y los resultados serán la más completa confirmación de mis promesas"’

\subsection{Estructura y metodología del Método Natural}

Piferrer inicia la obra con una serie de máximas de escritores y filósofos para convencerse y convencer al lector de que su idea de la enseñanza-aprendizaje de las lenguas modernas es la mejor. Parte de Duclos ("Pocas reglas, muchas reflexiones, y sobre todo mucha práctica, ved ahí la llave de todas las artes"), para seguir con Locke ("Las lenguas no se hicieron por reglas ó por arte, sino por el uso común de las gentes"), continuar con Sicard ("No puede uno aprender la gramática de una lengua, sea la que fuere, sino cuando ya sabe hablar, cuando ya sabe conversar"), y termina el propio Piferrer ("He observado que los que mejor saben las reglas, no siempre son los que hablan una lengua extranjera con más facilidad").

Metodológicamente plantea la estructura de la obra en tres tratados de casi igual número de páginas: sesenta y cuatro el primero, sesenta y cuatro el segundo, y sesenta y siete el tercero. El primero tiene veinticinco lecciones, el segundo veintiocho y dieciocho el tercero. El ritmo de las unidades o lecciones es en el primer tratado de poco más de una página, ritmo que irá cambiando según avance el aprendizaje del inglés. El tiempo que dedica a cada unidad es de noventa minutos, tiempo que el autor estima más que suficiente para dominar la materia que en ella se expone. La metodología empleada es la propia de la época: gramática-traducción. Pero con un planteamiento muy particular ya que Piferrer parte de elementos que se consideran básicos en este método de enseñanza-aprendizaje, pero que él descarta. Elimina el característico listado de palabras para el aprendizaje memorístico del vocabulario. Elimina la clásica regla de gramática que preside todas las lecciones del método gramática-traducción y que suele servir de base científica para el estudio de la lengua. Deja de lado el método deductivo en el que a través del estudio de reglas gramaticales se pretende capacitar al alumno para construir cualquier frase en la nueva lengua. También abandona la traducción inversa, frases generalmente construidas artificialmente con el fin de que en ellas queden reflejadas las reglas gramaticales que se han explicado con anterioridad, que él sustituye por la interlineal como ejercicio bipolar de aprendizaje. Y, sorprendentemente, un método que no se preocupa para nada de la pronunciación, ya que el objetivo no es aprender a hablar una lengua sino estar preparado para disfrutar de los textos escritos, se convierte en Piferrer en

7 Ibídem, p.7. 
un método donde la fonética es el eje central de las todas las unidades de aprendizaje del libro.

Las unidades de estudio del primer tratado son breves, poco más de una página. La finalidad de su enseñanza es que se aprenda de memoria el texto por la práctica repetitiva de los ejercicios, en el que las palabras se memorizan en su contexto. La estructura interna de cada una de las lecciones sigue el mismo ritmo: título (tema fonemático a estudiar), texto inglés, pronunciación, traducción, traducción interlineal y teoría. Los tres primeros ritmos aparecen en tres columnas paralelas.

El primer tratado, Lecturas, tiene a la fonética como columna vertebral de las veinticinco unidades de estudio. Y, aunque no lo diga el autor, la fonética será más tarde el lazo de unión y consistencia de los otros dos tratados. El segundo lo dedica al estudio de los verbos y a lo que él llama principios gramaticales. Para terminar con el estudio detallado de la sintaxis inglesa a la que dedica el tercer tratado en su totalidad.

El planteamiento metodológico de las lecciones del segundo y tercer tratado sigue la misma pauta del primero: título (tema gramatical a estudiar), lectura, pronunciación y traducción, traducción interlineal y teoría gramatical. Sólo cambia el contenido de las lecturas que ahora corresponden a los conceptos gramaticales explicados en la teoría de cada unidad. Así la lección XVIII que trata del artículo, muestra frases en las que el artículo the aparece trece veces, y siempre en cursiva, para atraer la atención del estudiante y alentar su aprendizaje gramatical por la reflexión personal y análisis de las oraciones en que éste aparece, con el fin de que él mismo induzca las correspondientes reglas. Didácticamente, estas unidades de estudio están perfectamente diseñadas para conseguir el fin que Piferrer se propone en cada una de ellas. Sin estudiar reglas, llegar a definirlas a través del análisis reflexivo de los diferentes textos.

Las lecturas base de cada una de las unidades de trabajo del primer tratado, trozos selectos los llama Piferrer, resultan ser una serie de sentencias, máximas o dichos (seis o siete por cada lección) de contenido ético y moral y, con frecuencia, altamente religioso. El texto original inglés es bueno y elegante. La traducción española, que aparece en la tercera columna, no es mala, en cuanto versión de los citados dichos o máximas. Simplemente va por otros caminos de la simple traducción directa. La interlineal, al traducir palabra a palabra, resulta extraña y artificial como texto castellano. No es buena como tal, pero al pretender que sirva para conseguir una fácil transferencia inglés-español, español-inglés con el fin de memorizarlas y que sirva al mismo tiempo de aprendizaje de vocabulario, la versión no se corresponde con el refrán o sentencia española. Pensamos que Piferrer explicaría a los alumnos esta más que notable disfunción. Presentamos unos ejemplos tomados al azar. En cursiva aparece el texto inglés. Debajo, la versión española. A continuación, la traducción interlineal.

When the fox preaches, beware of the geese.

Cuando el diablo reza, engañar quiere.

Cuando el zorro predica, tened cuidado de los gansos.

This lady has a fine little lapdog.

Esta señora tiene un hermoso perro de faldas.

Esta señora tiene un hermoso pequeño faldas-perro. 
Otro problema que hemos detectado en cuanto al aprendizaje del idioma se refiere, es que se aprecia en los textos ingleses que Piferrer ha seleccionado como modelos éticos, morales y religiosos, que carecen de cualquier tipo de diseño previo, programación y gradación de dificultades. Las lecturas del primer tratado son mucho más difíciles que las del segundo o el tercero. Estas últimas sorprenden por su sencillez.

\subsection{Técnicas de transcripción fonética}

Al analizar el Método Natural del autor catalán, nos ha sorprendido los planteamientos que diseña y la didáctica que aplica para sistematizar la enseñanza de la fonética inglesa a los españoles y hacerla lo más fácil y asequible posible a sus discípulos. Sabía que ésta era un área problemática en la adquisición del inglés, debido al desajuste permanente entre ortografía y pronunciación, fuente constante de problemas. Con ello pretendía desterrar de entre éstos "la vulgar y rancia preocupación que en su contra les domina: Preocupación hija de la infundada creencia de que la lengua inglesa es dificilísima en su pronunciación, dificilísima por el número de sus reglas y, en fin, dificultosa para ser hablada, para ser escrita y para ser leída".

Creemos que la pronunciación del inglés a finales de la primera mitad del siglo XIX no se diferenciaba mucho de la de hoy. Sin embargo, pensamos que las más que notables y llamativas variaciones que se observan al comparar la pronunciación de muchas palabras según el sistema diseñado por Piferrer y el IFA (Alfabeto de Fonética Internacional) o el IPA (International Phonetic Alphabet) que hoy se emplea ${ }^{9}$, se deben en gran medida al proceso de adaptación de los sonidos ingleses a unas pronunciaciones figuradas, diseñadas para ayudar a los estudiantes españoles a aproximarse a esa pronunciación, utilizando los recursos fónicos del español y del francés, lengua entonces más en boga entre las clases cultas. Recursos todos ellos muy limitados para la amplia gama de sonidos que presenta el idioma inglés. Así lo confirmaba Eduardo Benot (1851) cuando insistía en que sería el colmo del charlatanismo pretender representar exactamente la pronunciación del inglés con las figuraciones que en esa obra se utilizaban. En efecto, sólo se pretendía anunciar el poco más o menos de un sonido, pero nunca la igualdad. Y animaba a los alumnos que quisieran conseguir ésta a acudir a las clases de un nativo, ya que no había ni hay otra solución.

El planteamiento fonográfico que utiliza Piferrer no es difícil, ni complicado. Necesita sólo un buen maestro conocedor de las técnicas que guíe al alumno. Decimos esto porque, fiel al principio que guía toda su actuación lingüística (primero la práctica y luego la teoría), inicia la unidad primera sin indicación alguna del sistema de pronunciación figurada que va a utilizar, ni de las bases metodológicas en que se apoya, ni de los principios teóricos que lo sustentan. Y es que, de igual forma que abandonó el camino común de los maestros de lenguas europeos en lo que a la enseñanza-aprendizaje del idioma se refiere (mucha teoría y poca práctica), también dejó de lado el sistema numérico y de símbolos empleado por los lingüistas de entonces para enseñar la pronunciación de los diferentes idiomas.

${ }^{8}$ Anna Mountifield en Prefacio al Novísimo método teórico, práctico, analítico y sintético de Lengua Inglesa, de William Mountifield (1854). Madrid, p.5.

${ }^{9} \mathrm{Su}$ objetivo es intentar representar de modo gráfico la información transmitida oralmente, facilitando la captación de los hechos de habla con relativa exactitud 
Conocido es que durante el siglo XIX, y debido al gran desarrollo que experimentaron la fonética, la fisiología y la acústica, surgieron en Europa varios sistemas notacionales que gozaron de gran predicamento, como el llamado Alfabeto Estándar de Lepsius ${ }^{10}$, o los intentos por conseguir un alfabeto universal de Pitman ${ }^{11}$, Ellis ${ }^{12}$, Alexander Melville Bell ${ }^{13}$ o Henry Sweet ${ }^{14}$. Los trabajos de estos fonetistas fueron la base para la creación en 1888 de un sistema internacional de signos que sería llamado alfabeto de fonética internacional (AFI). En efecto, en esa fecha la Association Phonétique Internationale (API) decidió aunar los sistemas de notación que entonces se venían utilizando para representar el modo oral de las lenguas. Un grupo de profesores franceses, encabezados por el fonetista Paul Passy, decidió crear un sistema notacional capaz de resolver las dificultades que para sus alumnos suponía el alfabeto inglés. A este primer movimiento pronto se sumaron fonetistas como Henry Sweet, Otto Jespersen, Alexander Melville Bell, Max Müller, J. L. Lunden, A. H. Sayce y E. Sievers, que aportaron una visión más universal al proyecto francés. En este intento de la Asociación Internacional de Fonética se hallaban ya los dos principios básicos de la transcripción o representación fonética: respeto a los valores originales de las grafías latinas y un símbolo un fonema. Un alfabeto basado en la notación romana, completado con otras letras y signos diacríticos. Alfabeto de la Asociación que se irá revisando con el paso de los años como así ha sucedido en 1914, 1947 y 1969.

La obra de Francisco Piferrer, Método Natural (1846), es anterior a todos estos intentos por conseguir poner un orden en los diferentes sistemas de notación que se estaban utilizando para enseñar la pronunciación inglesa a los extranjeros. De aquí que siga su propio camino y utilice el sistema que se había diseñado y que desconocemos su origen. Sin aviso, ni explicación previa, aparece la lectura de la lección primera transcrita en notación figurada, utilizando la grafía romana con algún que otro símbolo de la fonética francesa. Estas son las primeras frases del texto transcrito por Piferrer:

${ }^{10}$ Karl Richard Lepsius fue un importante egiptólogo alemán. Nació en Naumburg en 1810 y falleció en Berlín en 1884. Fue autor de notables investigaciones sobre las lenguas del oriente antiguo. Durante cuatro años (1842-1846) exploró Egipto con Bunsen. Entre sus obras cabe destacar: El libro de los muertos (1842) y Monumentos de Egipto y Etiopía (1849-1859). El Alfabeto Estándar que ideó Lepsius fue un modelo muy utilizado por los misioneros para codificar las lenguas africanas y amerindias.

${ }^{11}$ Sir Isaac Pitman fue un notable estenógrafo británico. Nació en Trowbridge en 1813, y murió en Eath en 1897. Creó un alfabeto, el Phonotype (1876), y a él se debe la invenci ón del sistema de taquigrafía que lleva su nombre.

${ }^{12}$ Robinson Ellis fue un importante lingüista británico. Nació en Barning (Kent) en 1834 y falleció en Oxford en 1913. A parte de sus estudios y proyectos fonéticos (ideó un alfabeto, el Glossic, para la enseñanza de las lenguas extranjeras, y el Palaeotype para estudios filológicos y dialectológicos) es conocido por sus estudios sobre Cátulo.

${ }^{13}$ Alexander Melville Bell, padre del inventor del teléfono, escribió la obra Visible Speech, the Science of Universal Alphabetics (1867) con el objetivo de impartir una mejor enseñanza de la pronunciación del inglés tanto a nativos como a extranjeros.

${ }^{14}$ El libro de A. M. Bell ejercería una profunda influencia en el fonético británico H. Sweet, filólogo británico, nacido en Londres en 1845 y fallecido en Oxford en 1912. Sus principales obras son: Ensayo sobre la poesía anglosajona (1871), Nueva gramática Inglesa (1892-1989), e Historia del lenguaje (1890). 
Be prudent to-day, it would be madness to defer.

Bi prúdent tu-dé, it ud be mádnes tu difer.

Frequent good company, if you would keep a good name.

Fricuént gud cómpani, if yu kíp e gud ném.

Un primer análisis de la notación fonémica de Piferrer pone de manifiesto la castellanización prácticamente total de vocales y consonantes, la curiosa pronunciación de ciertas palabras $(t u-d e ́, u d)$ y la igualación de todas las vocales, no existe cantidad ni calidad en la representación vocálica. Es a partir de la unidad segunda en la que empieza a introducir sistemáticamente un método de pronunciación fonémica y una metodología didáctica para su enseñanza y aprendizaje. Antes dijimos que la fonética era la columna vertebral de todas las lecciones del primer tratado. Y, en efecto, así es. Bajo el título: Lección II, aparece una A mayúscula, para indicarnos que ésta será la vocal objeto de estudio y aprendizaje fónico a nivel segmental, de toda la unidad. Y en torno a este sonido girará toda la lección. Analizando el reducido texto, vemos que la grafía $a$ aparece veintidós veces, aunque sólo en ocho ocasiones lleva el sonido de $a^{15}$. Termina la unidad de estudio con un extenso tratado fonético en que presenta las posibles pronunciaciones del sonido $a$, su figuración y las múltiples combinaciones de la $a$ con otras vocales ${ }^{16}$. Exposición teórica que nos parece excesiva para principiantes en la lengua inglesa. Excesiva por la cantidad de reglas teóricas que presenta, del material aportado, y por el número de palabras que emplea y que resultan de poca o nula utilización frecuente. Como extraño y curioso es el modo de nominar y clasificar las distintas vocales con sonido propio. El mismo esquema se utiliza en las restantes lecciones del tratado primero. Por ejemplo, la lección III, que estudia la vocal y el sonido $e$, incluye treinta y una palabras que llevan incorporada dicha vocal $e^{17}$, a lo que sigue una extensísima teoría sobre la pronunciación de la $e$, en todas sus posiciones, excepciones y palabras individuales ${ }^{18}$. Y así con las

\footnotetext{
${ }^{15}$ Magnificence, calamity, regard, and, impart, adore, abstain, are.

${ }^{16}$ La $a$ inicia cinco diptongos (ai, ay, ao, au, aw) y dos triptongos (awe, aye).

$A i$, ay: suena como la a cerrada: Airiness (érines).

Ao: suena también como la $a$ cerrada: gaol (gél).

$A u$, aw: suena como a grave: maul, paw (môl, pô). Au suena como a española en aunt (ant).

Aye: se pronuncia éi: gayety (guéiti).

Awe: se pronuncia como ô grave: awe $(\hat{o})$.

${ }^{17}$ Honesty, are, very, commendable, believe, convenient, seem, be, indeed, goodness, evidently, appear, every, one, content, evidence, itself, commend, every, esteem, felicity, never, be, permanent, consider, life, like, garden, nettles, violets, flowers.

${ }^{18}$ Por excepción la $e$ suena $a$ española en clerk (clark).

La $e$ inicia siete diptongos:ea, ee, ei, ey, eo, eu, ew, y cuatro triptongos: eau, eye, eou, ewe.

ea: (i) beam (bim); (e) bread (bred); (a) heart (hárt); (é) bear (bér); (ioe) fear (fioer).

ee: (i) leek (lik)

ei: (é) feint (fent); (i) deceit (disít);

ey: (é); (i) key (ki).

eo: (i) people (pipol); (o) George (gorge); (e) Leopard (lépœrd);( $)$ pigeon (pigœn);(yú)

feodal (fyúdal).

eu, ew: (yú) sew (syú); (u) crew (cru).

eau: (o): bureau (biúro); (iú): beauty (biúti).

eou: suena como iœe.
} 
quince primeras unidades del primer tratado en que va estudiando minuciosamente las vocales y las consonantes inglesas. Dedica una lección a la pronunciación del alfabeto inglés. Las restantes estudian el acento.

\subsection{El alfabeto inglés}

Aunque Piferrer no inicia el estudio de la fonética con una explicación previa del alfabeto inglés, nosotros sí lo hacemos porque creemos que es necesario debido a la utilización que el autor hace a lo largo de todo el primer tratado de múltiples términos referidos al alfabeto. Así, cuando habla de la vocales alfabéticas, castellanas, oscuras, regulares, inglesas $o$ valonas, pronunciación de determinadas letras, de diptongos y triptongos propios e impropios, etc.

Dedica la lección XVI de la primera parte, posterior al tratado de la substancia fónica de vocales y consonantes, a estudiar el alfabeto inglés y su pronunciación aproximativa o más general. En la parte teórica de la unidad afirma que el alfabeto inglés consta de veintiséis letras, que se dividen en vocales y consonantes. Las vocales son siete: ' $a, e, i, o, u, w, y$; estas dos últimas son a veces consonantes.

Define luego diptongos y triptongos. Términos que más tarde divide en propios e impropios. Diptongos propios constan de dos sonidos distintos, como oi, ow. Los impropios son los que no tiene sino un sonido, como ae, ai. Algunos lingüistas les dan el nombre de vocales compuestas. Los triptongos propios constan de tres sonidos, como uoi; los que constan de menos de tres sonidos se llaman triptongos impropios, y también vocales triples ${ }^{19}$.

Las consonantes se dividen en simples y compuestas. Las primeras constan de un solo carácter, como $c, b, d$. Las compuestas constan de más de uno, como $c h$, th, etc. Hay en inglés diez consonantes compuestas, que son $c h, g h, g m, g n, p h, r h, s h, s s, t h$ y $w h$.

Letras y su pronunciación aproximativa:

\begin{tabular}{|c|c|c|c|c|c|c|c|c|c|}
\hline $\begin{array}{l}\mathrm{A}, \\
e ́\end{array}$ & $\begin{array}{l}\mathrm{B}, \\
b i,\end{array}$ & $\begin{array}{l}\mathrm{C}, \\
s i,\end{array}$ & $\begin{array}{l}\mathrm{D}, \\
d i,\end{array}$ & $\begin{array}{l}\mathrm{E}, \\
i,\end{array}$ & $\begin{array}{l}\mathrm{F}, \\
\text { ef, }\end{array}$ & $\begin{array}{c}\mathrm{G}, \\
\text { dché, }\end{array}$ & $\begin{array}{l}\mathrm{H}, \\
\text { etch, }\end{array}$ & $\begin{array}{l}\mathrm{I}, \\
a ́ i,\end{array}$ & $\begin{array}{c}\mathrm{J}, \\
d c h i,\end{array}$ \\
\hline $\begin{array}{l}\mathrm{L}, \\
e l\end{array}$ & $\begin{array}{l}\mathrm{M}, \\
\mathrm{em},\end{array}$ & $\begin{array}{l}\mathrm{N}, \\
\text { en, }\end{array}$ & $\begin{array}{l}\mathrm{O}, \\
\vartheta\end{array}$ & $\begin{array}{l}\mathrm{P}, \\
\text { pi, }\end{array}$ & $\begin{array}{l}\mathrm{Q}, \\
\text { quiú, }\end{array}$ & $\begin{array}{l}\mathrm{R}, \\
a r,\end{array}$ & $\begin{array}{l}\mathrm{S}, \\
e s,\end{array}$ & $\begin{array}{l}\mathrm{T}, \\
t i,\end{array}$ & $\begin{array}{l}\mathrm{U}, \\
i u ́,\end{array}$ \\
\hline $\begin{array}{l}\text { W, } \\
\text { cebliú }\end{array}$ & $\begin{array}{l}\mathrm{X}, \\
e k s,\end{array}$ & $\begin{array}{c}\mathrm{Y}, \\
\text { uái, }\end{array}$ & $\begin{array}{c}\mathrm{Z} . \\
\text { set. }\end{array}$ & & & & & & \\
\hline
\end{tabular}

Vocales:
A, E, I, O, U, Y, W.
e, i, ái, $\quad$, iú, uai, dobliú.

${ }^{19}$ Dos o tres vocales juntas dejan de formar diptongo o triptongo, cuando alguna de ellas requiere una emisión de aire particular, un nuevo esfuerzo de voz, lo que generalmente sucede cuando dicha vocal lleva el acento, como en zoology, que tiene el acento en la segunda o, por cuyo motivo las dos oo se pronuncian separadamente sin formar diptongo. 
Diptongos y su pronunciación más general:

\begin{tabular}{|c|c|c|c|c|c|}
\hline $\begin{array}{c}\mathrm{AE}, \\
i\end{array}$ & $\begin{array}{c}\mathrm{AI}, \\
e ́,\end{array}$ & $\begin{array}{c}\mathrm{AY}, \\
e ́,\end{array}$ & $\begin{array}{c}\mathrm{AO}, \\
e ́,\end{array}$ & $\begin{array}{c}\mathrm{AU}, \\
\hat{o},\end{array}$ & $\begin{array}{c}\mathrm{AW}, \\
\hat{o},\end{array}$ \\
\hline $\begin{array}{c}\text { EA, } \\
i,\end{array}$ & $\begin{array}{c}\mathrm{EE}, \\
i,\end{array}$ & $\begin{array}{c}\mathrm{EI}, \\
e ́,\end{array}$ & $\begin{array}{c}\mathrm{EY}, \\
e ́,\end{array}$ & $\begin{array}{c}\mathrm{EO}, \\
\alpha,\end{array}$ & $\begin{array}{c}\text { EU, } \\
i u ́,\end{array}$ \\
\hline $\begin{array}{l}\text { IA, } \\
\text { áia, }\end{array}$ & $\begin{array}{c}\mathrm{IE}, \\
i,\end{array}$ & $\begin{array}{c}\mathrm{IO}, \\
\alpha,\end{array}$ & & & \\
\hline $\begin{array}{c}\mathrm{OA}, \\
\nabla,\end{array}$ & $\begin{array}{c}\mathrm{OE}, \\
\diamond,\end{array}$ & $\begin{array}{l}\text { OI, } \\
\hat{o} i,\end{array}$ & $\begin{array}{c}\mathrm{OY}, \\
\hat{o} i,\end{array}$ & $\begin{array}{c}\mathrm{OO}, \\
u,\end{array}$ & $\begin{array}{c}\mathrm{OU}, \\
\hat{o} u,\end{array}$ \\
\hline $\begin{array}{l}\mathrm{UA}, \\
\text { ué, }\end{array}$ & $\begin{array}{l}\mathrm{UE}, \\
\text { ué, }\end{array}$ & $\begin{array}{l}\text { UI, } \\
u i,\end{array}$ & $\begin{array}{l}\text { UY, } \\
u i,\end{array}$ & $\begin{array}{c}\text { UO. } \\
u .\end{array}$ & \\
\hline
\end{tabular}

Triptongos y su pronunciación

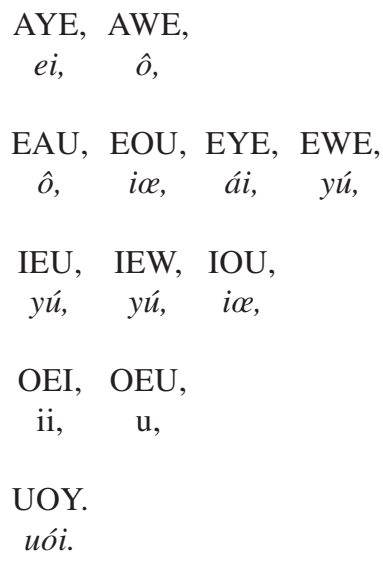

\subsection{Representación de las vocales}

Partiendo de la idea clave de la enseñanza que promueve Piferrer (primero la práctica y luego la teoría), tenemos que ir de atrás hacia delante, unidad tras unidad, para obtener la organización metodológica y didáctica del sistema de pronunciación que él diseñó, primero de las vocales y luego de las consonantes, cómo se representan o figuran y, a continuación, cómo se pronuncian.

Piferrer distingue 20 sonidos vocálicos ingleses diferenciados. Sin embargo, Walker en su Dictionary de 1849 no encuentra más que 14, y que representa por vocales numeradas:

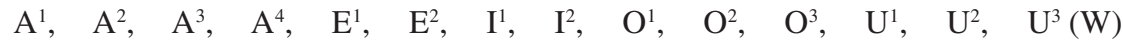


Para Lindley Murray (1844) no existen más que diez sonidos vocálicos, tras analizar las opiniones más generalizadas de los gramáticos de su tiempo ${ }^{20}$.

Piferrer distingue tres especies de $a$ : abierta, cerrada y grave.

$$
\begin{aligned}
& \text { a abierta se figura como a } \\
& \text { a cerrada se figura como é } \\
& \text { a grave se figura como } \hat{o}
\end{aligned}
$$

La a abierta se pronuncia como en español: regard (rigárd).

La a cerrada suena como la $e$ castellana en la voz té: mates (méts).

La $a$ abierta se pronuncia entre $a$ y $o$, participando más de esta última: all (ôl).

Recuerda que estos tres sonidos pueden estar acentuados o no. De ahí resulta el desacuerdo de los gramáticos en lo que a esta letra se refiere. Así, unos le dan cuatro sonidos, otros cinco, y algunos hasta seis.

Como regla general, afirma que existen cuatro especies de $e$ :

e castellana: se figura $e$

e inglesa: se figura $i$

e oscura: se figura $x$

e muda: se figura como - , o nada

La $e$ castellana suena como en español: bed (bed).

La $e$ inglesa suena como $i$ castellana: belief (bilif).

La $e$ oscura suena entre $a$ y $e$, algo más clara que la $e u$ francesa: term (torm).

La $e$ muda carece de sonido y se suprime en la pronunciación: scene $(\sin )$.

Según Piferrer existen tres especies de $i / y$.

$i$ castellana: se figura $i$

$i$ inglesa: se figura ái

i oscura: se figura $e$

La $i$ castellana suena como en español: vanity (vániti).

La inglesa se pronuncia ai: silence (sáilens).

La $i$ oscura tiene el mismo sonido que la $e$ oscura: virtue (vœrtchíu).

Lo dicho de la $i$ se aplica, hasta cierto punto, a la $y$.

Seis son las especies de $o$ que enumera y representa figurativamente.

o alfabética: se figura $\nabla^{21}$,

$o$ grave: se figura $\hat{\text {, }}$

$o$ regular, se figura como la $o$ española

o oscura: se figura $\alpha$,

o cerrada: se figura $u$,

o muda: carece de figuración.

20 "It appears, that there are in the English Language fourteen simple vowels; but as $i$ and $u$ when pronounced long may be considered as diphthongs, or diphthongal vowels, our Language, strictly speaking, contains but twelve simple vowels; to represent which, we have only 5 distinct characters or letters. If $a$ in far is the same specific sound as $a$ in fat; and $u$ in bull, the same as $o$ in move, which is the opinion of some grammarians; there are but ten original vowel sounds in the English Language" (Lindley Murray, Grammar of the English Language. London, p.16).

${ }^{21}$ Es el símbolo más aproximado a la $o$ hecha con trazos góticos que hemos encontrado en el ordenador. 
La o alfabética se pronuncia disponiendo los órganos de la voz como para pronunciar $u$, sacando los labios extraordinariamente fuera; mas al instante de expeler el aire, sin mudar en nada la posición de la boca, pronúnciese' $o$, y resultará precisamente el sonido de la $o$ alfabética, que es igual al de la vocal compuesta francesa $a u$, como en la voz so $(s \emptyset)$.

La $o$ grave se pronuncia abriendo la boca en ademán de pronunciar $a$, mas al instante de expeler el aire, sin mudar en nada la disposición de los órganos, se procura articular $o$, como en la voz sorry (sôrri).

La o regular se pronuncia como la o castellana, flock (flok).

La o oscura se pronuncia disponiendo la boca para pronunciar $e$, y procurando sin mudarla de esta disposición, pronunciar $o$. Lo que da lugar al sonido de la $o$ oscura, que es casi igual al de la $e$ y de la $i$ oscuras, como se ve en las voces above (abœv), wisdom (uísdom).

La o cerrada se pronuncia como la $u$ castellana, así, to move (tu muv).

La $o$ muda carece de sonido y se suprime enteramente en la pronunciación figurada o se la sustituye con (-).

Piferrer diseña cuatro especies de $u$ :

$u$ alfabética: se representa por iú

u cerrada: se figura por la $u$ española

u oscura: se figura por $\propto$

$u / w$ valona: se figura por $\mathrm{v}$

La $u$ alfabética se pronunica iú: cubic (kiúbik),

La $u$ cerrada suena como en español: bull ( $b u l)$, full ( $f u l)$.

La $u$ oscura suena como las demás vocales oscuras, y se figura del mismo modo, but (bœt).

La $u$ o $w$ valona es una vocal que suena casi como la $u$ castellana. Se dice casi, porque al expeler el aire para pronunciar $u$, se hace con algún esfuerzo que le da cierto roce con la $v$ de los valencianos. Para formarse una idea de este sonido, es menester esforzarse en articular la sílaba $b u$ sin cerrar los labios, were (vér).

\subsection{Representación de las consonantes}

En el tratamiento y representación de las consonantes Piferrer sigue la misma estructura metodológica y principios didácticos que empleó con las vocales. Analicemos la lección VII que trata de la consonante $h$ y la propondremos como modelo para todas las demás. En primer término aparece bajo el número de la lección una $h$, para indicar que ésta será la vocal que vertebrará toda la lección. En el texto de la lectura aparecen quince palabras que llevan incorporada la consonante $h$. La teoría se dedica exhaustivamente a estudiar la pronunciación de la $h$ y la variada casuística que genera.

Piferrer distingue dos tipos de h:

$h$ aspirada: se figura con el símbolo $\mathrm{H}$

$h$ muda: no tiene pronunciación figurada

La $h$ aspirada suena en inglés como una especie de $j$ muy suave, del mismo modo que la pronuncian comúnmente los andaluces en las vocales que comienzan por la sílaba hue, como huevo, hueso. Ejemplos: husband (Hœsband), have (Hav). 
La $h$ muda, como su nombre indica, se calla enteramente y se suprime en la pronunciación figurada, honesty (ónesti).

De entre las consonantes compuestas con $h$, destaca por su interés y dificultad para los alumnos las siguientes: $p h, s h, t h$.

$$
p h \text { se figura como } f \text { y se pronuncia como la } f \text { española. }
$$

Sin embargo, en las palabras compuestas de una que acaba en $p$ y de otra que principia por $h$, cada una de estas letras conserva su pronunciación peculiar: shepherd (cHép-Hoerd), porque es una voz compuesta de shep y herd. En las voces que siguen sólo se pronuncia la $p$ : diphthong, naphtha, ophthalmy, triphthong, y derivados. La $p h$ es muda en apophthegm, phthisic, y derivados. En nephew (néviu) y Stephen (stevn) la ph suena como $v$.

sh se figura $c H$

La sh se pronuncia como la $c h$ de los franceses. Para dar una idea de este sonido, afirma Piferrer lo siguiente: "traslado aquí lo que digo acerca de él en mi Método para aprender el francés, la $c h$ francesa se pronuncia como en castellano, sin más diferencia que apoyar la lengua en los dientes de abajo, poniendo particular cuidado en no tocar con ella a ninguna parte del paladar, resultando un sonido algo gutural. Para indicarlo nos valemos de este carácter $c h$, y a favor de los que conozcan aquellas lenguas, debemos decir que la $c h$ francesa es exactamente la sh de los ingleses, la $x$ de los catalanes y la sch de los alemanes".

La th se figura $z$ (sonido duro) $y d z$ (sonido suave).

La th tiene un sonido muy fuerte o duro, parecido a la $z$ española, y otro suave, que se forma de este modo. "Sepárense los labios, y sáquese la lengua como cosa de medio dedo fuera de la boca, y retirándola ligeramente, haciéndola rozar con los dientes de arriba, procúrese pronunciar $z a, z e$, ze \&; la articulación que resulta es exactamente la de la th suave. Convengo en que el gesto a que obliga esta operación no es de los más graciosos, pero no se ha de perder de vista lo siguiente: $1^{\circ}$ que haciendo ese gesto, es muy fácil pronunciar la th suave, cuando de otro modo es muy difícil si no imposible dar con dicho sonido; $2^{\circ}$ este gesto se hace sólo al principio, mas una vez obtenido el sonido, se repite o reproduce fácilmente y sin casi pensar en ello"22.

La $c h$ se figura como ch castellana

Como regla general, $c h$ se pronuncia como en español.

La $g$ se figura $g$ (sonido fuerte) y $d c h$ (sonido suave)

La $g$ se pronuncia fuerte como la $g$ española en la palabra garganta y otro suave como el de la $j$ francesa precedida de una especie de $d$. Para articular este sonido, aplíquese la circunferencia de la lengua a los dientes de arriba en todo su contorno, como para pronunciar

${ }^{22}$ Francisco Piferer, ob., cit., p.1-26. 
$d$, y expeliendo el aire con fuerza, procúrese articular simultáneamente $d$ e $y$, así $d-y a, d$-ye, $d-y o, d-y u$. Resultarán aproximadamente las sílabas inglesas $j a, j e, j i, j o$, ju, que se figurarán por scha, dche, dchi, dcho, dchu.

$c$ se figura $c$ (sonido fuerte) y $s$ (sonido sonoro)

$k$ se figura $k$ y tiene uniformemente el sonido de la $c$ fuerte

La $c$ se pronuncia fuerte, como en español, antes de $a, o, u$, como en can (can), catch (catch), could $(c u d)$ y al final de sílaba o dicción. Se pronuncia sonora, igual al de la $s$ castellana, antes de las vocales $e, i, y$.

$$
\begin{aligned}
& l \text { se figura } l \\
& l l \text { se figura } l l
\end{aligned}
$$

La $l$ se pronuncia como en español: flies (fláis). La lengua inglesa carece de sonido de $l l$ castellana. De modo que dos $l l$ reunidas se pronuncian generalmente como una sola: kills (kils), full (ful), y rara vez se hacen sentir separadamente las dos.

$$
\begin{gathered}
m \text { se figura } m \\
n \text { se figura } n
\end{gathered}
$$

La $m$ y la $n$ se pronuncian como en castellano: milman (milman), dinner (dínær).

$$
\begin{aligned}
& b \text { se figura } b \\
& p \text { se figura } p
\end{aligned}
$$

La b y la p se pronuncian como en español, base (bés), person (persn).

$$
r \text { se figura } r
$$

La $r$ se pronuncia generalmente como en español: morning (môrning), care (kér), tomorrow (tu-môrr $\downarrow$ ).

$$
\begin{gathered}
s \text { se figura } \\
s \text { (sonido fuerte castellano) } \\
s \text { se figura } \\
s \text { (cursiva) (sonido suave de } z \text { francesa) } \\
t \text { se figura } t \\
z \text { se figura } z
\end{gathered}
$$

La s tiene el sonido fuerte español, makes (méks), store (st $\diamond \mathrm{r}$ ). Respecto al sonido de $s$ suave o z francesa, afirma Piferrer que trastada aquí lo que dijo acerca de este sonido en el Idioma francés al alcance de todos, lectura, lección VI, pág. 15: “... y es el más complicado del alfabeto. Bastan los labios para pronunciar $b, p, m$; con la lengua y los dientes formamos los sonidos $d$, $t$; con la misma y el paladar producimos $c h$ y $l$; pero en la $z$ concurren la lengua, los dientes, el paladar, la nariz, el gaznate; y es por consiguiente lingual, dental, palatal, nasal y gutural. Excusado nos parece añadir que letra tan complicada debe oírse de viva voz; sin embargo, para articularla sin dificultad, pronúnciese suavemente la combinación ags, no haciendo más que tocar la $s$ tan ligeramente como sea posible, y sin esforzar la voz ni mudar los órganos de la posición que toman para pronunciar ags, prolónguese este sonido, produciendo como un silbido débil y suave, parecido al susurro de la abeja, y concluyendo 
con una vocal, resultarán las voces ags-á, ags-é, ags-í, ags-ó, ags-ú, cuya última parte $s$-á, $s$-é, s-í, s-ó, s-ú, equivaldrá a los sonidos franceses za, zé, zi, zo, zu, zou".

La $t$ se pronuncia, por regla general, como en castellano.

La $z$ tiene, por regla general, el sonido de $z$ francesa o $s$ suave, como queda explicado.

\subsection{El acento}

Dedica Piferrer cuatro lecciones (XVII, XVIII, XVIX y XX) a estudiar el acento en inglés. Acento en las palabras, que no en la oración. Acento que él expresa gráficamente.

Partiendo de un texto sobre Alejandro Magno y su espíritu generoso en la lucha contra Darío de Persia, expone su teoría sobre el concepto de acento y la cantidad, tipos de acento, composición de las sílabas y sus clases, comparando sus valoraciones con el castellano y otras lenguas, en especial el francés y el alemán. En español se confunde el acento con la cantidad, de modo que toda sílaba acentuada se considera como larga. En inglés, como en la mayor parte de las lenguas, son dos cosas muy distintas, pudiendo una sílaba estar acentuada sin ser larga, o ser larga sin estar acentuada. Se entiende por cantidad el espacio más o menos largo de tiempo que se emplea en pronunciar una sílaba.

El genio de la lengua inglesa en cuanto al acento se aparta enteramente de las demás lenguas. Recuerda Piferrer que en latín hay tres acentos: el agudo ', que designa una elevación de tono, el grave " para designar una disminución del mismo, y cuando era menester elevar y bajar la voz en una misma sílaba, se valían del acento circunflejo, figurado así ^ . En inglés no hay ningún acento escrito, pero toda palabra o voz de más de una sílaba se pronuncia con acento, es decir elevando o apoyando la voz en una sílaba más que en las otras de la misma palabra. Advierte que hay palabras que tienen dos y hasta tres acentos, en cuyo caso uno se llama acento principal, y los demás secundarios. Este acento es algo más débil que el principal, y tiene por objeto, no tanto evitar la monotonía que resultaría de muchas sílabas pronunciadas en un mismo tono, como el sostener la voz y contribuir a la distinción de los sonidos.

Con respecto al número de sílabas, se da el nombre de monosílaba a la palabra de una sola sílaba, disílaba es la que consta de dos sílabas, trisílaba la que tiene tres, tetrasílaba la de cuatro, pentasílaba la de cinco, y polisílaba la que consta de más de cinco sílabas.

Dedica en su totalidad la lección XVIII a tratar el acento en las disílabas, la XIX, al estudio del acento en las trisílabas y la XX, al acento de las polisílabas. Termina el tema del acento en las apalabras con una advertencia a los alumnos: No debe perderse de vista que estas reglas son generales, y están sujetas por tanto a muchas, quizá demasiadas, excepciones. En esta unidad da por terminada toda la teoría que se puede ofrecer a los alumnos sobre la fonética. Las lecciones XXI, XXII, XXIII y XXIV están dedicadas a ejercicios de diálogos $\mathrm{y}$, finalmente, en la lección XXV y última del primer tratado hace un extenso repaso (siete páginas de letra menuda) de cuantas normas y reglas se han ido citando en la parte teórica de las veintitrés primeras lecciones. Repasa las vocales y consonantes y hace referencia a las unidades en que se trataron estos sonidos. 


\section{Metodología de la enseñanza de la pRonunciación}

Como antes afirmamos, no deja de ser sorprendente que Piferrer se sirva de un método de enseñanza-aprendizaje del tipo gramática y traducción (en el que no existe preocupación alguna por la pronunciación) para diseñar un sistema de enseñanza en el que la pronunciación sea el eje central de las todas las unidades de aprendizaje del libro. Para lograr su objetivo parte de un minucioso diseño estructural de la unidad de estudio. Inicia el trabajo llamando la atención del alumno sobre el tema fónico a tratar en la lectura con el fin de centrar en ese sonido toda su atención. Sonido que suele escribir en cursiva. Luego en los cuatro ritmos restantes de la unidad (texto inglés - pronunciación - traducción - traducción interlineal) va practicando esos contenidos fónicos hasta el punto de hacerlos consustanciales con el alumno por la repetición constante de palabras y sonidos. En lecciones breves (poco más de una página), con tiempo más que suficiente (noventa minutos) y con una práctica repetitiva de los ejercicios (en los que las palabras se memorizan en su contexto) va consiguiendo una buena enseñanza-aprendizaje de la pronunciación del inglés. La última parte de cada unidad, la fijación teórica y memorística de los temas estudiados (reglas generales y excepciones) resulta fácil y efectiva. Y es que parecen cosas ya vistas, analizadas y practicadas. Llegar a la inducción de reglas será algo natural y sencillo. Por último, la lección que dedica al final del tratado primero al repaso general de las reglas aportadas en cada una de las lecciones, es una prueba más del permanente control que hace el autor de la buena pronunciación del alumno. Buena pronunciación que seguirá buscando a lo largo de los otros dos tratados.

Piferrer ha dejado consignados los pasos que maestro y discípulo deben seguir para conseguir el éxito que el autor persigue con su plan. En primer término aconseja al maestro leer repetidas veces, las que considere necesarias de acuerdo con la situación de aprendizaje del grupo o alumno, el texto consignado bajo la columna pronunciación (la que está en el medio de las tres que presenta la lectura). Lectura que deben repetir luego los propios discípulos en forma individualizada. En este primer paso es básica la acción del maestro que guiará primero al alumno en todo momento a una correcta pronunciación de la lengua y le introducirá en el sistema de notación o pronunciación figurada que ha ideado Piferrer, y corregirá luego las posibles desviaciones fónicas.

Dominada la lectura de la pronunciación, invitará al discípulo a leer la columna del texto, procurando hacerlo exactamente como indica la columna de pronunciación. El siguiente paso será leer atentamente, y repetidas veces, la traducción interlineal, hasta que puedan decirse las palabras inglesas en español, y aún las españolas en inglés. En este punto radica la base de un aprendizaje dinámico y significativo del vocabulario integrado en un contexto. Para luego volver a leer alternativamente el texto y la traducción, repitiendo este ejercicio hasta que pueda traducirse el primero en español, y aún reproducirlo en inglés con sólo mirar la traducción. Para terminar el ejercicio copiando el texto inglés, procurando escribirlo tantas veces como sea necesario, hasta llegar también a reproducirlo correctamente por escrito con sólo mirar la traducción, aprendiendo de este modo simultánea y progresivamente a leer, traducir, hablar y escribir.

Otra de las estrategias que utiliza para la enseñanza de la pronunciación es la del acercamiento permanente a los sonidos del español para dar confianza y seguridad a los discípulos al contar con un punto de referencia seguro. Es constante la referencia a "se pronuncia como en español", "suena como $e$ castellana", "suena como en español", "suena como $i$ castellana", "se pronuncia como lao castellana", " $u$ cerrada suena como en español", 
"suena casi como la $u$ castellana", "se pronuncia como la $f$ española", "parecido a la $z$ española", "se figura como ch castellana", "se pronuncia fuerte como la $g$ española en la palabra garganta", "se pronuncia fuerte como en español", "la $l$ se pronuncia como en español", "la $m$ y la $n$ se pronuncian como en castellano", "la $b$ y la $p$ se pronuncian como en español", "la $s$ tiene el sonido fuerte español", "la $t$ se pronuncia, por regla general, como en castellano", etc. Por supuesto que este elemento positivo de confianza y seguridad del discípulo tiene su parte negativa en la castellanización de los signos representativos de la pronunciación inglesa. Las notaciones de vocales aparecen iguales, con la misma cantidad y calidad. No existen vocales breves ni largas. Todas están equiparadas a las cinco españolas. Propone un sistema de pronunciación en el que todas las palabras están individualizadas, puras, como viene en los diccionarios, sin conexión alguna con los otros componentes de la cadena hablada. Esto, por supuesto, sólo ocurre en los listados de palabras de los textos de enseñanza-aprendizaje del inglés o en los diccionarios. Los acentos de las palabras aisladas al integrarse en una frase, se subordinan al acento de dicha frase, perdiendo parte de su estructura fónica en beneficio de la tonalidad y la comunicación. Para Piferrer estas formas no existen, todos los vocablos conservan su estructura fonética.

Y da un paso más en esta estrategia de acercamiento a los sonidos del castellano intentando dar detalles de cómo posicionar los órganos de la voz para emitir determinados sonidos, ocho exactamente (o alfabética, o grave, o oscura, $u$ valona, sh, th, $g$ y $z$ ), de complicada pronunciación para los alumnos españoles. Así, "la o alfabética se pronuncia disponiendo los órganos de la voz como para pronunciar $u$, sacando los labios extraordinariamente fuera; mas al instante de expeler el aire, sin mudar en nada la posición de la boca, pronúnciese' $o$, y resultará precisamente el sonido de la $o$ alfabética, que es igual al de la vocal compuesta francesa $a u$, como en la voz so $(s \downarrow)$ )"; "la o grave se pronuncia abriendo la boca en ademán de pronunciar $a$, mas al instante de expeler el aire, sin mudar en nada la disposición de los órganos, se procura articular o, como en la voz sorry (sôrri); "la o oscura se pronuncia disponiendo la boca para pronunciar $e$, y procurando sin mudarla de esta disposición, pronunciar $o$. Lo que da lugar al sonido de la $o$ oscura, que es casi igual al de la $e$ y de la $i$ oscuras, como se ve en las voces above (abøv), wisdom (uísdom); "la $u$ o $w$ valona es una vocal que suena casi como la $u$ castellana. Se dice $c a s i$, porque al expeler el aire para pronunciar $u$, se hace con algún esfuerzo que le da cierto roce con la $v$ de los valencianos. Para formarse una idea de este sonido, es menester esforzarse en articular la sílaba $b u$ sin cerrar los labios, were (vér); "la sh se pronuncia como la ch de los franceses. Para dar una idea de este sonido, afirma Piferrer lo siguiente: "traslado aquí lo que digo acerca de él en mi Método para aprender el francés, la ch francesa se pronuncia como en castellano, sin más diferencia que apoyar la lengua en los dientes de abajo, poniendo particular cuidado en no tocar con ella a ninguna parte del paladar, resultando un sonido algo gutural. Para indicarlo nos valemos de este carácter $c h$, y a favor de los que conozcan aquellas lenguas, debemos decir que la $c h$ francesa es exactamente la $s h$ de los ingleses, la $x$ de los catalanes y la $s c h$ de los alemanes"; "la th tiene un sonido muy fuerte o duro, parecido a la $z$ española, y otro suave, que se forma de este modo: "Sepárense los labios, y sáquese la lengua como cosa de medio dedo fuera de la boca, y retirándola ligeramente, haciéndola rozar con los dientes de arriba, procúrese pronunciar $z a, z e$, ze \&; la articulación que resulta es exactamente la de la th suave. Convengo en que el gesto a que obliga esta operación no es de los más graciosos, pero no se ha de perder de vista lo siguiente: $1^{\circ}$ que haciendo ese gesto, es muy fácil pronunciar la th suave, cuando de otro modo es muy difícil si no imposible dar con dicho sonido; $2^{\circ}$ este gesto se 
hace sólo al principio, mas una vez obtenido el sonido, se repite o reproduce fácilmente y sin casi pensar en ello"; "la $g$ se pronuncia fuerte como la $g$ española en la palabra garganta y otro suave como el de la $j$ francesa precedida de una especie de $d$. Para articular este sonido, aplíquese la circunferencia de la lengua a los dientes de arriba en todo su contorno, como para pronunciar $d$, y expeliendo el aire con fuerza, procúrese articular simultáneamente $d$ e $y$, así $d-y a, d-y e, d-y o, d-y u$. Resultarán aproximadamente las sílabas inglesas $j a, j e, j i, j o, j u$, que se figurarán por scha, dche, dchi, dcho, dchu"; "pero en la $z$ concurren la lengua, los dientes, el paladar, la nariz, el gaznate; y es por consiguiente lingual, dental, palatal, nasal y gutural. Excusado nos parece añadir que letra tan complicada debe oírse de viva voz; sin embargo, para articularla sin dificultad, pronúnciese suavemente la combinación ags, no haciendo más que tocar la $s$ tan ligeramente como sea posible, y sin esforzar la voz ni mudar los órganos de la posición que toman para pronunciar ags, prolónguese este sonido, produciendo como un silbido débil y suave, parecido al susurro de la abeja, y concluyendo con una vocal, resultarán las voces ags-á, ags-é, ags-í, ags-ó, ags-ú, cuya última parte $s$-á, s-é, s-í, $s$-ó, s-ú, equivaldrá a los sonidos franceses za, zé, zi, zo, zu, zou".

Como resulta obvio, no se olvida de la viva voz del maestro como guía necesaria para una buena pronunciación. En especial cuando los sonidos se complican y resulta imprescindible su voz de referencia y su experiencia.

Otra estrategia de enseñanza-aprendizaje que utiliza es el mantenimiento de los mismo cinco ritmos de presentación de contenidos en todas las unidades de trabajo de los tres tratados. Se mantiene fijo desde la primera unidad a la última. Esta uniformidad proporciona seguridad y confianza al profesor y facilita al alumno el estudio.

Aunque la estructura y el desarrollo de las unidades de trabajo las encontramos metodológicamente correctas en la consecución de su objetivo (primero la práctica y luego la teoría), pensamos que la parte que cada unidad dedica a la teoría es exagerada y por lo mismo no muy didáctica. Exagerada por el cúmulo de excepciones que va citando a las reglas de pronunciación que explica y comenta. Creemos que cita todos los casos que existen y conoce. Alguna siempre está bien para que el alumno se vaya familiarizando con ellas. Tantas, pensamos que le desbordan y le llevan a cuestionarse las reglas, y mucho más aún para los que se inician el aprendizaje del inglés.

\section{Conclusiones}

Considero que el estudio de los libros de texto, en este caso, El Idioma Inglés puesto al alcance de todos o Método Natural para aprender el inglés de un modo fácil y agradable, sin cansar la memoria, y personalidades como Piferrer son fundamentales no sólo para poder conocer qué se estaba haciendo a nivel metodológico y práctico a mediados del XIX respecto a la enseñanza de la pronunciación inglesa, sino también para que los docentes del área conozcan los planteamientos y metodologías que se pusieron en marcha para intentar solventar unas carencias que como hemos podido comprobar a la luz de los resultados que sistemáticamente distintos organismos como el INECSE en Vez y Martínez (2002), e investigaciones López de Blas (2000) ponen de relieve un hecho, cuando menos preocupante, como que los propios profesionales de la enseñanza admiten que la competencia comunicativa oral es a la que prestan menos tiempo en sus clases. Carencias que no se circunscriben simplemente al siglo XIX sino que se han repetido durante el XX y se están constatando en el XXI. 
Queda mucho camino por recorrer en la mejora de la enseñanza de la pronunciación inglesa, pero trabajos como los del lingüista catalán nos dan una visión histórica que ayuda a los docentes a entender su trabajo y observar con otros ojos la realidad en la que están inmersos y, lo que es más importante, nos invita a reflexionar sobre lo que está sucediendo en las aulas. Me permito terminar lanzando al lector el desafío -reflexión del autor: "es escasísimo el número de los que llegan, no diré a hablar, mas ni aun a leer medianamente el francés o el inglés. Hace ya muchos años que tan extraña anomalía llamó mi atención, induciéndome naturalmente a investigar la causa de tan desagradable resultado".

\section{Bibliografía}

Benot, E. (1851): Nuevo Método del Dr. Ollendorff para aprender a leer, hablar y escribir una lengua cualquiera adaptado al inglés por Eduardo Benot, obra calculada para aprender este idioma en seis meses, seguida de un apéndice, y acompañada, en volumen separado, de las claves de los temas y de un diccionario que por el orden de lecciones contiene todas las palabras y frases enseñadas en el texto, y la indicación de su prosodia. Revisada la parte inglesa por George Knowles Shaw. Cádiz: Imprenta, librería y litografía de la Revista Médica.

Buenaventura, D. (1983): "Los libros de texto como fuente para la Historia de la Educación", en Historia de la Educación 2: 353-358.

Callamand, M. (1981): Méthodologie de l'enseignement de la prononciation. Paris: Cecle International. Casey, G. (1819): Gramática Inglesa para españoles. Barcelona: Francisco Piferrer.

Casey, G. (1849): Diccionario de la Pronunciación Crítica de la Lengua Inglesa. Barcelona: V. Torras y J. Corominas.

Gabriel de, N. \& Viñao, A. (1999).'La investigación histórico-educativa. Barcelona: Methodica.

Gil, Escudero, G. \& Alabau Valcells, I. (1997). Evaluación comparada de la enseñanza y aprendizaje de la lengua inglesa. Madrid: INECSE.

López de Blas, M. (2000). "Changing perceptions". In'English Teaching Professional, 17:59-60.

Martín-Gamero, S. (1961): La enseñanza del inglés en España: desde la Edad Media hasta el siglo $X X$. Madrid: Gredos.

Monroy, R. (1998): Sistemas de transcripción fonética del inglés: teoría y práctica. Granada: Grupo Editorial Universitario.

Ovilo y Otero, M. (1859). Manual de Biografía y Bibliografía de los escritores del siglo XIX. Tomo II. París: Librería de Rosa y Bouret.

Palau y Dulcet, A. (1923-27). Manual del librero hispanoamericano. 7 vols. Barcelona: LA.

Piferrer, F. (1847). El Idioma Inglés puesto al alcance de todos o Método Natural para aprender el inglés de un modo fácil y agradable, sin cansar la memoria. Madrid: Casimiro Monier.

Vez, J.M. y Martínez Piñeiro, E. (eds.) (2002). Competencia comunicativa oral en lenguas extranjeras. Investigación sobre logros del alumnado gallego de inglés y francés al finalizar la ESO. Santiago de Compostela: I.C.E. de la Universidad de Santiago de Compostela.

Vilar, M. (2004). Docentes, traductores e intérpretes de la lengua inglesa en la España del siglo XIX: Juan Calderón, los hermanos Usoz y Pascual de Gallangos. Murcia: Serv. De publicaciones del al Universidad de Murcia.

Viña Rouco, Mar (2005). "Metodología inductiva y deductiva en la enseñanza de las lenguas vivas en España en el siglo XIX". Porta Linguarum 4. Granada: GEU, pp.185-200.

Villoria, J. (2007). "Metodología y didáctica de la enseñanza de la pronunciación inglesa en España: José de Urcullu (1825)". En prensa. 